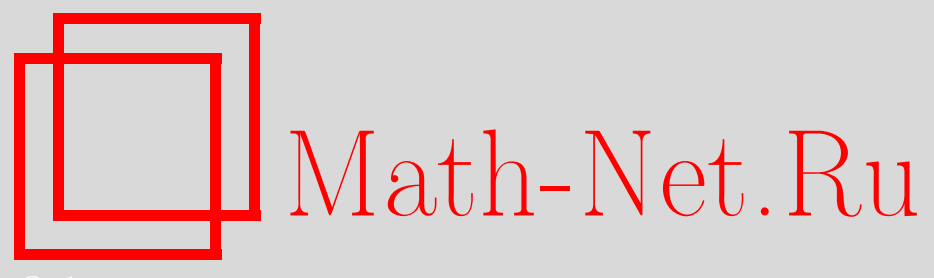

С. С. Мирзоев, И. Дж. Джафаров, О разрешимости одной краевой задачи для операторно-дифференциальных уравнений второго порядка в частных производных, $M a-$ тем. заметки, 2012, том 91, выпуск 3, 470-472

DOI: https://doi.org/10.4213/mzm9320

Использование Общероссийского математического портала Math-Net.Ru подразумевает, что вы прочитали и согласны с пользовательским соглашением http://www . mathnet.ru/rus/agreement

Параметры загрузки:

IP: 3.85 .5 .30

26 апреля 2023 г., 14:23:23

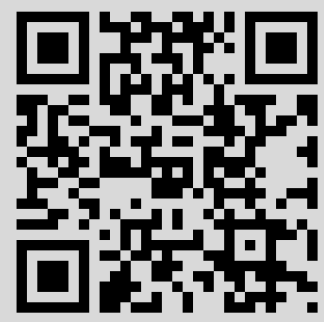




\section{О разрешимости одной краевой задачи для операторно-дифференциальных уравнений второго порядка в частных производных}

\section{С. С. Мирзоев, И. Дж. Джафаров}

Пусть $H$ - сепарабельное гильбертово пространство, $A$ - нормальный обратимый оператор в $H$. Тогда оператор $A$ имеет полярное разложение $A=U C$, где $U$ - унитарный, а $C$ - положительно-определенный самосопряженный операторы в $H$. Обозначим через $H_{\gamma}(\gamma \geqslant 0)$ шкалу гильбертовых пространств, порожденную оператором $C$, т.е.

$$
H_{\gamma}=D\left(C^{\gamma}\right), \quad(\varphi, \psi)_{\gamma}=\left(C^{\gamma} \varphi, C^{\gamma} \psi\right), \quad \varphi, \psi \in D\left(C^{\gamma}\right), \quad \gamma \geqslant 0 .
$$

При $\gamma=0$ считаем, что $H_{0}=H$.

Пусть $t \in \mathbb{R}_{+}=(0,+\infty), x \in \mathbb{R}=(-\infty,+\infty)$ и положим $\mathbb{R}_{+}^{2}=\mathbb{R}_{+} \times \mathbb{R}$. Рассмотрим в $\mathbb{R}_{+}^{2}$ следующую краевую задачу:

$$
\begin{gathered}
-\frac{\partial^{2} u}{\partial t^{2}}-\frac{\partial^{2} u}{\partial x^{2}}+A^{2} u+A_{1,0} \frac{\partial u}{\partial t}+A_{0,1} \frac{\partial u}{\partial x}+A_{1,1} \frac{\partial^{2} u}{\partial t \partial x}+A_{0,0} u=f(t, x),(t, x) \in \mathbb{R}_{+}^{2}, \\
u(0, x)=0,
\end{gathered}
$$

где $f(t, x), u(t, x)$ - функции, определенные почти всюду в $\mathbb{R}_{+}^{2}$, со значениями в $H$, а операторные коэффициенты удовлетворяют следующим условиям:

а) $A$ - нормальный обратимый оператор, спектр которого содержится в угловом секторе

$$
S_{\varepsilon}=\left\{\lambda:|\arg \lambda| \leqslant \varepsilon, 0 \leqslant \varepsilon<\frac{\pi}{2}\right\}
$$

b) $B_{1,0}=A_{1,0} A^{-1}, B_{0,1}=A_{0,1} A^{-1}, B_{1,1}=A_{1,1}, B_{0,0}=A_{0,0} A^{-2}$ суть ограниченные операторы в $H$.

Обозначим через $L_{2}\left(\mathbb{R}_{+}^{2} ; H\right)$ гильбертово пространство измеримых $H$-значных функций $f(t, x)$, для которых

$$
\|f\|_{L_{2}\left(\mathbb{R}_{+}^{2} ; H\right)}=\left(\int_{\mathbb{R}_{+}^{2}}\|f(t, x)\|^{2} d t d x\right)^{1 / 2}<+\infty .
$$

Через $D\left(\mathbb{R}_{+}^{2} ; H_{2}\right)$ обозначим линеал, состоящий из бесконечно дифференцируемых функций с компактным носителем в $[0,+\infty) \times \mathbb{R}$. Определим пространство $W_{2}^{2}\left(\mathbb{R}_{+}^{2} ; H\right)$ как пополнение $D\left(\mathbb{R}_{+}^{2} ; H_{2}\right)$ по норме

$$
\|u\|_{W_{2}^{2}\left(\mathbb{R}_{+}^{2} ; H\right)}=\left(\sum_{j=0}^{2-k} \sum_{k=0}^{2}\left\|C^{2-(k+j)} \frac{\partial^{k+j} u}{\partial t^{k} \partial x^{j}}\right\|_{L_{2}\left(\mathbb{R}_{+}^{2} ; H\right)}^{2}\right)^{1 / 2} .
$$

Обозначим

$$
\stackrel{\circ}{W}_{2}^{2}\left(\mathbb{R}_{+}^{2} ; H\right)=\left\{u: u(t, x) \in W_{2}^{2}\left(\mathbb{R}_{+}^{2} ; H\right), u(0, x)=0\right\} .
$$

Очевидно, этот линеал является собственным подпространством в $W_{2}^{2}\left(\mathbb{R}_{+}^{2} ; H\right)$. При выполнении условия $\mathrm{b})$ для функций $u(x, t) \in W_{2}^{2}\left(\mathbb{R}_{+}^{2} ; H\right)$ каждое слагаемое в правой части уравнения (1) корректно определено и является функцией из $L_{2}\left(\mathbb{R}_{+}^{2} ; H\right)$. Пусть правая часть $f \in L_{2}\left(\mathbb{R}_{+}^{2} ; H\right)$. Функцию $u(x, t) \in W_{2}^{2}\left(\mathbb{R}_{+}^{2} ; H\right)$ будем называть регулярным решением уравнения (1), если равенство (1) выполняется почти при всех $t>0$. 
ОПределЕниЕ. Задачу (1), (2) назовем регулярно разрешимой, если для любой функции $f(t, x) \in L_{2}\left(\mathbb{R}_{+}^{2} ; H\right)$ существует функция $u(x, t) \in \dot{W}_{2}^{2}\left(\mathbb{R}_{+}^{2} ; H\right)$, которая является регулярным решением уравнения (1), причем имеет место оценка

$$
\|u\|_{W_{2}^{2}\left(\mathbb{R}_{+}^{2} ; H\right)} \leqslant \text { const }\|f\|_{L_{2}\left(\mathbb{R}_{+}^{2} ; H\right)},
$$

где постоянная не зависит от $f$.

В данной работе мы укажем достаточные условия, которые обеспечивают регулярную разрешимость задачи (1), (2). Эти условия выражаются только коэффициентами операторно-дифференциального уравнения (1). Отметим, что в одномерном случае разрешимость начально-краевых задач исследована многими авторами (см., например, [1]-[8]), в двумерном же случае, когда $A$ - самосопряженный положительно-определенный оператор - в работе [9]. На всей плоскости разрешимость уравнения (1) рассмотрена, например, в работах [10]-[12].

Обозначим через

$$
\begin{array}{ll}
P_{0} u=-\frac{\partial^{2} u}{\partial t^{2}}-\frac{\partial^{2} u}{\partial x^{2}}+A^{2} u, & u(t, x) \in \stackrel{\circ}{W}_{2}^{2}\left(\mathbb{R}_{+}^{2} ; H\right), \\
P_{1} u=A_{1,0} \frac{\partial u}{\partial t}+A_{0,1} \frac{\partial u}{\partial x}+A_{1,1} \frac{\partial^{2} u}{\partial t \partial x}+A_{0,0} u, & u(t, x) \in \stackrel{\circ}{W}_{2}^{2}\left(\mathbb{R}_{+}^{2} ; H\right) .
\end{array}
$$

Сначала исследуем регулярную разрешимость задачи

$$
\begin{aligned}
P_{0} u=-\frac{\partial^{2} u}{\partial t^{2}}-\frac{\partial^{2} u}{\partial x^{2}}+A^{2} u & =f(t, x), \quad(t, x) \in \mathbb{R}_{+}^{2}, \\
u(0, x) & =0 .
\end{aligned}
$$

\section{Имеет место}

Теорема 1. Пусть выполняется условие а). Тогда задача (3), (4) регулярно разрешимa.

Из этой теоремы следует, что оператор $P_{0}: \stackrel{\circ}{W}_{2}^{2}\left(\mathbb{R}_{+}^{2} ; H\right) \rightarrow L_{2}\left(\mathbb{R}_{+}^{2} ; H\right)$ является изоморфизмом. Поэтому в пространстве $\stackrel{\circ}{W}_{2}^{2}\left(\mathbb{R}_{+}^{2} ; H\right)$ нормы $\left\|P_{0} u\right\|_{L_{2}\left(\mathbb{R}_{+}^{2} ; H\right)}$ и $\|u\|_{W_{2}^{2}\left(\mathbb{R}_{+}^{2} ; H\right)}$ эквивалентны.

Справедлива

Теорема 2. Пусть выполняется условие а). Положим

$$
\begin{aligned}
& c_{0}(\varepsilon)=\left\{\begin{array}{lll}
1 & n p u & 0 \leqslant \varepsilon \leqslant \pi / 4, \\
1 / \sin 2 \varepsilon & n p u & \pi / 4<\varepsilon<\pi / 2,
\end{array}\right. \\
& c_{1}(\varepsilon)=\frac{1}{2 \cos \varepsilon} \quad n p u \quad 0 \leqslant \varepsilon<\frac{\pi}{2}, \\
& c_{2}(\varepsilon)=\left\{\begin{array}{lll}
1 & n p u & 0 \leqslant \varepsilon \leqslant \pi / 4, \\
1 / \sqrt{2} \cos \varepsilon & \text { npu } & \pi / 4<\varepsilon<\pi / 2 .
\end{array}\right.
\end{aligned}
$$

Тогда при любом $и \in W_{2}^{2}\left(\mathbb{R}_{+}^{2} ; H\right)$ имеют место следующие оценки:

$$
\begin{gathered}
\left\|A^{2} u\right\|_{L_{2}\left(\mathbb{R}_{+}^{2} ; H\right)} \leqslant c_{0}(\varepsilon) c_{2}(\varepsilon)\left\|P_{0} u\right\|_{L_{2}\left(\mathbb{R}_{+}^{2} ; H\right)}, \\
\left\|A \frac{\partial u}{\partial t}\right\|_{L_{2}\left(\mathbb{R}_{+}^{2} ; H\right)} \leqslant c_{0}^{1 / 2}(\varepsilon) c_{1}(\varepsilon)\left\|P_{0} u\right\|_{L_{2}\left(\mathbb{R}_{+}^{2} ; H\right)},
\end{gathered}
$$




$$
\begin{gathered}
\left\|A \frac{\partial u}{\partial x}\right\|_{L_{2}\left(\mathbb{R}_{+}^{2} ; H\right)} \leqslant c_{1}(\varepsilon) c_{2}(\varepsilon)\left\|P_{0} u\right\|_{L_{2}\left(\mathbb{R}_{+}^{2} ; H\right)}, \\
\left\|\frac{\partial^{2} u}{\partial t \partial x}\right\|_{L_{2}\left(\mathbb{R}_{+}^{2} ; H\right)} \leqslant c_{0}^{1 / 2}(\varepsilon) c_{1}(\varepsilon)\left\|P_{0} u\right\|_{L_{2}\left(\mathbb{R}_{+}^{2} ; H\right)} .
\end{gathered}
$$

Из (5)-(8) получаем, что оператор $P \equiv P_{0}+P_{1}: \stackrel{\circ}{W}_{2}^{2}\left(\mathbb{R}_{+}^{2} ; H\right) \rightarrow L_{2}\left(\mathbb{R}_{+}^{2} ; H\right)$ ограничен.

В результате, можно установить достаточные условия регулярной разрешимости задачи (1), (2).

Теорема 3. Пусть выполняются условия а), b) и имеет место неравенство

$$
\alpha(\varepsilon)=c_{0}^{1 / 2}(\varepsilon) c_{1}(\varepsilon)\left(\left\|B_{1,0}\right\|+\left\|B_{1,1}\right\|\right)+c_{1}(\varepsilon) c_{2}(\varepsilon)\left\|B_{0,1}\right\|+c_{0}(\varepsilon) c_{2}(\varepsilon)\left\|B_{0,0}\right\|<1,
$$

где числа $c_{0}(\varepsilon), c_{1}(\varepsilon), c_{2}(\varepsilon)$ определяются в теореме 2. Тогда задача $(1),(2)$ регулярно разрешима.

Отметим, что условия теоремы 3 о регулярной разрешимости точны при каждом $\varepsilon \in$ $[0, \pi / 2)$.

СледствиЕ. Пусть $A$ - положительно-определенный самосопряженный оператор и имеет место неравенство

$$
\alpha=\frac{1}{2}\left(\left\|B_{1,0}\right\|+\left\|B_{0,1}\right\|+\left\|B_{1,1}\right\|\right)+\left\|B_{0,0}\right\|<1 .
$$

Тогда задача (1), (2) регулярно разрешима.

\section{СПИСОК ЦИТИРОВАННОЙ ЛИТЕРАТУРЫ}

[1] М. Г. Гасымов, Докл. АН СССР, 199:4 (1971), 747-750. [2] М. Г. Гасымов, С. С. Мирзоев, Дифферени. уравнения, 28:4 (1992), 651-661. [3] Ю. А. Дубинский, Матем. сб., 90:1 (1973), 3-22. [4] А. Г. Костюченко, А. А. Шкаликов, Функи. анализ и его прил., 17:2 (1983), 38-61. [5] С. С. Мирзоев, Докл. АН СССР, 273:2 (1983), 292-295. [6] А. А. Шкаликов, Тр. сем. им. И. Г. Петровского, 14, Изд-во Моск. ун-та, М., 1989, 140-224. [7] А. Р. Алиев, С. С. Мирзоев, Функи. анализ и его прил., 44:3 (2010), 63-65. [8] С. С. Мирзоев, Ф. А. Гулиева, Матем. заметки, 86:5 (2009), 797-800. [9] С. С. Мирзоев, Тр. ИММ НАН Азербайджана, 8 (1998), 154-161. [10] Г. И. Асланов, Международный семинар "Дифференциальные уравнения и смежные вопросы" (пятнадцатая сессия совместных заседаний семинара им. И.Г. Петровского и Московского математического общества 19-22 января 1993 года), УМН, 48:4 (1993), 172-173. [11] Г. И. Асланов, Докл. РАН, 337:1 (1994), 10-13. [12] Х. В. Ягубова, Вестн. Бакинского ун-та. Сер. физ.-матем. наук, 1998, № 3, 94-101.

С. С. Мирзоев

Бакинский государственный университет, г. Баку

E-mail: mirzoyevsabir@mail.ru

\section{И. Дж. Джафаров}

Институт математики и механики

НАН Азербайджана, г. Баку
Поступило 28.06.2011 